POS $\quad$ PROCEEDINGS

\title{
LLAGN and jet-scaling probed with the EVN
}

\section{Zsolt Paragi*}

Joint Institute for VLBI in Europe (JIVE), Postbus 2, 7990 AA Dwingeloo, The Netherlands

E-mail: zparagiejive.nl

\section{Zhi-Qiang Shen}

Shanghai Astronomical Observatory, CAS, 80 Nandan Road, Shanghai 200030, P.R. China Key Laboratory of Radio Astronomy, CAS, P.R. China

E-mail: zshen@shao.ac.cn

\section{Francesco de Gasperin}

University of Hamburg, Gojenbergsweg 112, D-21029, Hamburg, Germany

E-mail: stsf309@hs.uni-hamburg.de

\section{Jun Yang}

Joint Institute for VLBI in Europe (JIVE), Postbus 2, 7990 AA Dwingeloo, The Netherlands

E-mail: yang@jive.nl

\section{Andrea Merloni}

Max-Planck-Institut für Extraterrestrische Physik, Gießenbachstr. 85741 Garching, Germany Exzellenzcluster Universe, Boltzmann Str. 2, 85748 Garching, Germany

E-mail: am@mpe.mpg.de

\section{Zhi-Xuan Li}

Yunnan Astronomical Observatory, NAOC, 650011 Kunming, P.R. China

E-mail: lzx@ynao.ac.cn

Accreting black holes (BH) on all mass scales (from stellar to supermassive) appear to follow a nonlinear relation between X-ray luminosity, radio luminosity and $\mathrm{BH}$ mass, indicating that similar physical processes drive the central engines in X-ray binaries and active galactic nuclei (AGN). However, in recent years an increasing number of $\mathrm{BH}$ systems have been identified that do not fit into this scheme. These outliers may be the key to understand how BH systems are powered by accretion. Here we present results from EVN observations of a sample of low-luminosity AGN (LLAGN) with known mass that have unusually high radio powers when compared with their Xray luminosity.

11th European VLBI Network Symposium \& Users Meeting,

October 9-12, 2012

Bordeaux, France

\footnotetext{
* Speaker.
} 


\section{Introduction}

A deep physical understanding of the relations between accretion power and relativistic jet acceleration in active galactic nuclei (AGN) is a key goal of high-energy astrophysics. Recent progress in the field became possible by comparing AGN with stellar-mass X-ray binary systems (BHXRB or microquasars), carried out since the discovery of tight correlations between the Xray and radio luminosities in the so-called low-hard state of BHXRB [1]. In this spectral state microquasars produce a collimated, synchrotron self-absorbed compact jet [2] analogous to the flat spectrum radio cores seen in radio-loud AGN [3]. In an attempt to unify (hard state) stellar mass and (low-power) supermassive black holes, Merloni, Heinz \& di Matteo [4] and Falcke, Körding \& Markoff [5] independently found that BHXRB and low-luminosity AGN (LLAGN) occupy a plane in the three dimensional-space defined by the X-ray luminosity $\left(L_{X}\right)$, radio luminosity $\left(L_{R}\right)$ and black hole mass $M_{\mathrm{BH}}$, widely referred to as the fundamental plane of black whole activity (FP in the following).

The physics behind this is understood: these are all sub-Eddington accretors ( $L_{\mathrm{Edd}}<\sim 10 \%$ ) with radiatively inefficcient accretion flows, where $L_{X}$ is a measure of accretion rate and $L_{R}$ is a measure of the jet power, and both scale with $M_{\mathrm{BH}}$ in a predictable way [4, 5]. It comes as a natural idea to use the FP relations to get rough estimates of $\mathrm{BH}$ masses on various types of sources where $M_{\mathrm{BH}}$ is not known, but $L_{\mathrm{X}}$ and $L_{\mathrm{R}}$ are measured (e.g. [6]). One problem is that there is a significant scatter in the FP relations, and a few AGN classes simply do not fit. This issue was successfully addressed by more recent work [7,8], decreasing the scatter significantly. The AGN that do not fit either accrete at much higher rate than a few percent of the Eddington rate and may become more dominated by the accretion disc rather than the jet (e.g. bright quasars), or the measured $L_{\mathrm{X}}$ is subject to severe X-ray cooling (e.g. FR-I radio galaxies). However, there may be other factors that have to be taken into account. Notably, in AGN, there are no accretion disc states known that would directly correspond to the ones we see in microquasars. But with VLBI we can verify that the AGN emission is related to a self-absorbed compact jet (as in the case of BHXRB in the hard state), or it has resolved structure on milliarcsecond (mas) scales.

\section{The sample}

Recently, de Gasperin et al. [9] probed the black hole jet scaling relations in a sample of low-luminosity active galactic nuclei. They selected type 2 AGN from the Sloan Digital Sky Survey with $M \sim 10^{8} \mathrm{M}_{\odot}$ within the redshift range $z=0.05-0.11$, that are not located in a cluster environment. They observed the selected 17 LLAGN $\left(L_{X}<10^{42} \mathrm{erg} / \mathrm{s}\right)$ with the Chandra X-ray Telescope and the VLA in A-array configuration at $5 \mathrm{GHz}$ and $8.4 \mathrm{GHz}$. Sources from this sample were unresolved in the VLA FIRST survey at $1.4 \mathrm{GHz}$ with a resolution of 5 arseconds. At the higher resolution (330 mas and 230 mas at 5 and $8.4 \mathrm{GHz}$ with the VLA A-array, respectively) all the sources except one were still unresolved. The measured spectral indices range from very steep to steep, flat, and in one case inverted. Rather surprising, all sources had radio flux densities two-three orders of magnitude higher than expected from the FP relations for accreting black holes. The authors argued that the relation between the X-ray, OIII emission line and radio luminosities was indicative of a lack of molecular (torus-like) gas in these galaxies, and they proposed that the 

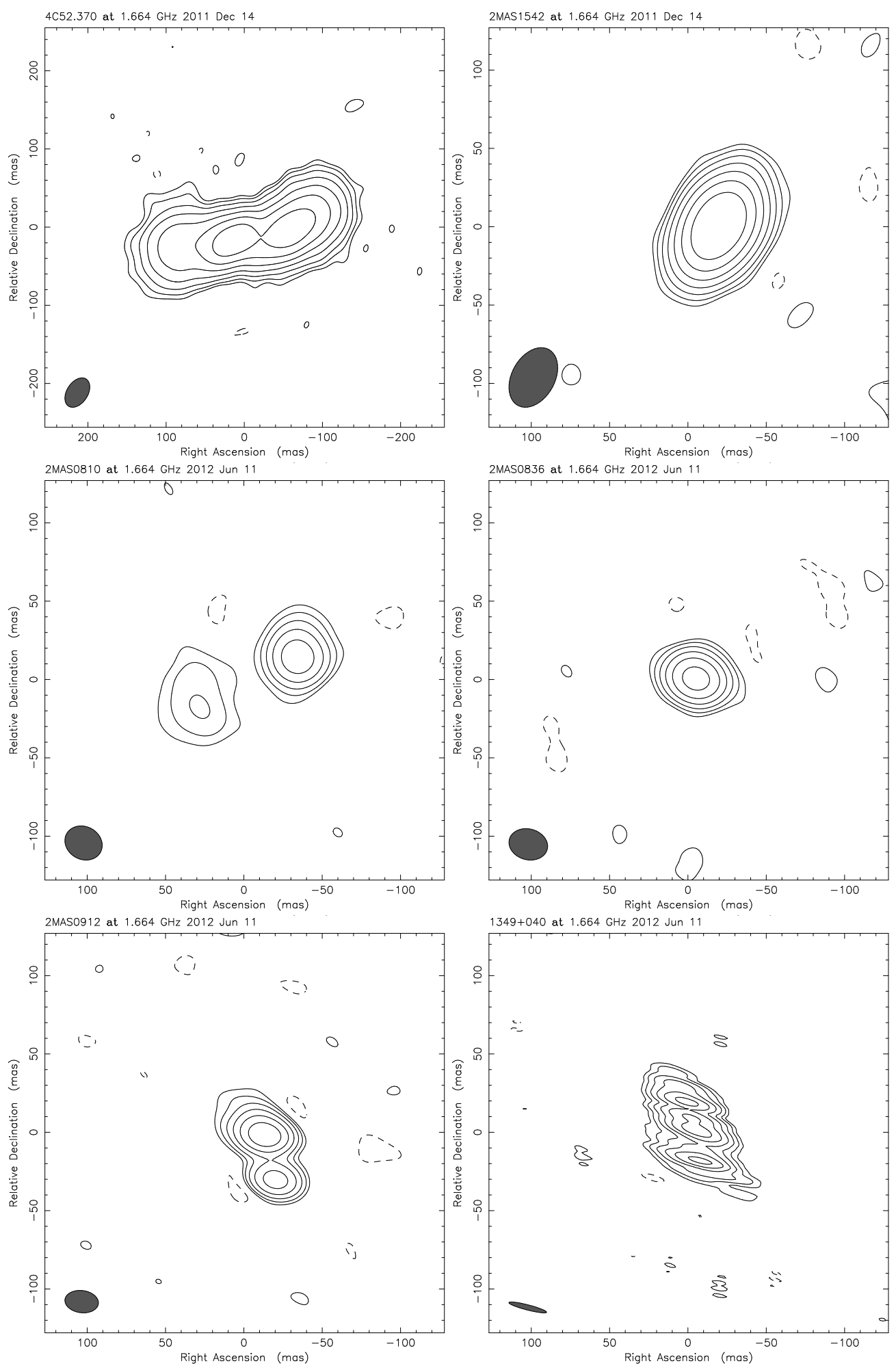

Figure 1: Naturally weighted e-EVN images of the sources. The contour levels are factors of two of the $3 \sigma$ image noise levels, which are 1.50, 0.5, 0.26, 0.75, 0.50, 1.0, 0.13, 1.0, 0.40 and $0.20 \mathrm{mJy} / \mathrm{beam}$ for our ten sources. 

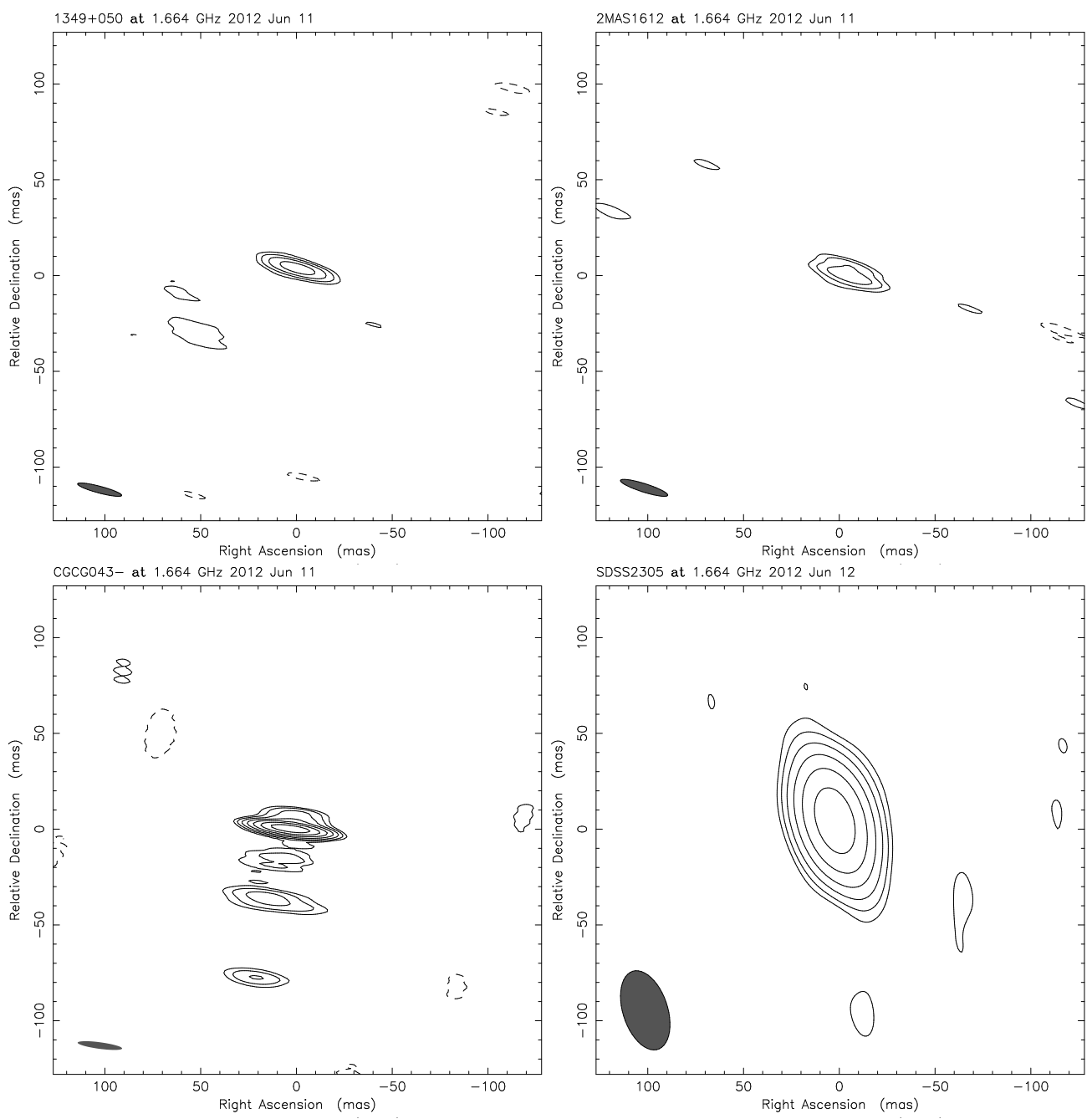

Figure 1: (continued)

accretion flow does not contribute to the observed X-ray emission, which instead is a tracer of the inner compact jet itself, likely differently from the main FP samples. This makes a good case for VLBI, to probe the inner jet structure and jet-accretion coupling in these LLAGN in massive early-type galaxies.

\section{EVN results}

We selected 10 of the sample sources that had total flux densities above $10.0 \mathrm{mJy}$ in the VLA FIRST survey, and observed them with the e-EVN at $1.6 \mathrm{GHz}$. The targets were phase-referenced to nearby calibators and they were all detected. The resulting images are shown in Fig. 1. The basic source parameters, the measured VLBI peak brightnesses and brightness temperatures, as well as the observed morphologies are listed in Table 1. Note that the brightness temperatures are not well constrained in most cases in this preliminary survey, because the only long baselines available were to Hartebeesthoek that could not observe the targets with very high declinations. 
Table 1: Basic source parameters and VLBI properties of our LLGN sample. The first five columns indicate the source name, redshift, X-ray luminosity $\log (L[\mathrm{erg} / \mathrm{s}])$, FIRST total flux density at $1.4 \mathrm{GHz}[\mathrm{mJy}]$, and radio spectral index $\left[S \propto v^{\alpha}\right]$ between 4.8 and $8.4 \mathrm{GHz}$ [9], while the last three columns indicate the VLBI peak intensity [mJy/beam], maximum brightness temperature at $1.6 \mathrm{GHz}\left[10^{9} \mathrm{~K}\right]$ and observed morphology, as inferred from this work.

\begin{tabular}{l|rrrr|rrr}
\hline Object & $z$ & $\begin{array}{r}\log L_{\mathrm{X}} \\
2-10 \mathrm{keV}\end{array}$ & $\begin{array}{r}S_{\mathrm{FIRST}} \\
1.4 \mathrm{GHz}\end{array}$ & $4.8-8.4 \mathrm{GHz}$ & $\begin{array}{r}I_{\mathrm{VLBI}} \\
1.6 \mathrm{GHz}\end{array}$ & $\begin{array}{r}T_{\mathrm{B}} \\
10^{9} \mathrm{~K}\end{array}$ & Morphology \\
\hline 4C52.370 & 0.11 & 41.7 & 575.70 & -0.94 & 103.0 & 0.06 & Resolved jet \\
2MAS1542+52 & 0.07 & 42.0 & 28.58 & +1.14 & 22.7 & $>0.29$ & Compact \\
2MAS0810+48 & 0.08 & 41.1 & 47.44 & -0.94 & 19.8 & 0.13 & CSO \\
2MAS0836+53 & 0.10 & 41.7 & 17.87 & -0.08 & 22.8 & $>0.72$ & Compact \\
2MAS0912+53 & 0.10 & 41.2 & 135.65 & -0.41 & 57.0 & 1.35 & CSO? \\
2MAS1349+04 & 0.08 & 40.4 & 63.31 & -1.04 & 6.2 & 0.2 & Resolved jet \\
2MAS1349+05 & 0.08 & 41.0 & 12.14 & -0.23 & 1.6 & $>0.02$ & Compact \\
2MAS1612+00 & 0.06 & 41.3 & 16.79 & -0.16 & 7.6 & $>1.82$ & Compact \\
CGCG043-05 & 0.07 & 43.2 & 70.03 & -0.17 & 43.2 & 8.68 & Core-jet \\
SDSS2308-09 & 0.10 & 41.3 & 15.71 & -0.10 & 9.6 & $>0.11$ & Compact \\
\hline
\end{tabular}

Five of the sources are compact on 10-mas scales (consistent with unresolved core-jets), and one shows a resolved core-jet structure. This latter source, CGCG043-05, was identified as a type 1 AGN after the initial sample was formed. Two sources show a resolved continuous jet on 10-100 mas scales without a strong radio core component, and two are (most likely) compact symmetric objects (CSO). On one hand, these results confirm the old VLBI wisdom that steep spectrum sources are resolved, flat spectrum sources are compact. It is interesting however to note that there are two very steep spectrum $(\alpha \sim-1)$ sources that are detected, and these have radio structures contained within a few hundred mas. New e-EVN results show that in some cases even ultra-steep spectrum sources $(\alpha<-1.4)$ have compact structure [10], therefore the steep spectrum source population should not be automatically excluded from VLBI studies of LLAGN samples.

Our target sources are LLAGN because their X-ray luminosity is below or equal to $10^{42} \mathrm{erg} / \mathrm{s}$, but they are actually quite bright in the radio. Based on the FP-relations, for a typical source in our sample with $L_{\mathrm{X}} \sim 10^{41} \mathrm{erg} / \mathrm{s}$ and $\mathrm{BH}$ mass of $\sim 10^{8} \mathrm{M}_{\odot}$, the X-ray to radio luminosity ratio should be $L_{\mathrm{R}} / L_{\mathrm{X}} \sim 10^{-3}$. As noted above, the observed radio luminosities are 2-3 orders of magnitude higher. From our EVN images we cannot draw a single conclusion to explain this radio loudness. Similarly to more luminous AGN classes, they have various structural properties on mas scales.

De Gasperin et al. [9] argued that there is no sign for significant absorption in the X-rays, that would cause the apparent low X-ray luminosity. Instead, they proposed that the X-ray emission may come from the jet base rather than the accretion flow. This idea was originally proposed for another LLAGN sample [11,12], with an even lower X-ray luminosity. We do see evidence for compact radio structure ( $60 \%$ of the sample) that could be consistent with this interpretation, although only in one case we can resolve a core-jet structure (the type 1 AGN). The remaining four sources are resolved on 10-100 mas scales and do not fit into this scenario.

For the extended sources there may be an alternative explanation. The radio structure is ob- 
served on $100 \mathrm{kpc}$ scales. In this case, the emission may be already decoupled from the AGN X-ray emission, since the latter is related to the more recent activity, while the earlier activity may be still imprinted in the radio emission we observe on larger scales. This is because of the long lifetime of relativistic electrons in the radio regime. This would be a straightforward explanation of the fact that steep spectrum sources do not fit on the fundamental plane of black hole activity. The flat spectrum sources in the sample are more difficult to explain. They have somewhat higher X-ray luminosity than expected for a radiatively inefficient accretion disc-driven jet system discussed by $[11,12]$. Doppler boosting of the radio emission is ruled out, because these are type 2 AGN. The only type $1 \mathrm{AGN}$ in the sample (CGCG043-05) has a brightness temperature that can be explained with a (slightly deboosted) equipartitional compact jet. We note also that this core-jet source has the lowest radio to X-ray luminosity ratio in the sample.

One may wonder why all members of a randomly-selected LLAGN sample appear brighter than expected in the radio. We note that this is partly a selection effect, because when we compare the typical $L_{\mathrm{X}} \sim 10^{41} \mathrm{erg} / \mathrm{s}$ of our sources with the detection threshold of FIRST in the redshift range of our sample, $L_{\mathrm{R}} \sim 10^{39} \mathrm{erg} / \mathrm{s}$, we get $L_{\mathrm{R}} / L_{\mathrm{X}} \sim 10^{-2}$. In this regard the fact that we see a number of FP outliers in our sample is not surprising.

\section{Conclusions}

We show that X-ray selected LLAGN that are compact on VLA scales can be well studied with VLBI regardless of their spectral index. We propose that the X-ray emission from the AGN and the large-scale radio emission may be decoupled in the extended, steep spectrum sources. The reason for the radio-loudness of compact sources remains a mistery.

\section{Acknowledgements}

The EVN is a joint facility of European, Chinese, South African and other radio astronomy institutes funded by their national research councils. ZP thanks Leonid Gurvits for comments.

\section{References}

[1] Corbel, S., Nowak, M.A., Fender, R.P. et al. 2003, A\&A, 400, 1007

[2] Dhawan, V., Mirabel, I.F., Rodríguez, L.F., 2000, ApJ 543, 373

[3] Blandford, R.D., Königl, A., 1979, A\&A, 232, 34

[4] Merloni, A., Heinz, S., di Matteo, T., 2003, MNRAS, 345, 1057

[5] Falcke, H., Körding, E., Markoff, S., 2004, A\&A, 414, 895

[6] Paragi, Z., Garrett, M.A., Biggs, A., 2007, PoS(MQW6)061

[7] Körding, E., Falcke H., Corbel, S., 2006, A\&A, 456, 439

[8] Plotkin, R.M., Markoff, S., Kelly, B.C. et al., 2012, MNRAS, 419, 267

[9] de Gasperin, F., Merloni, A., Sell, P. et al., 2011, MNRAS, 415, 2910

[10] Argo, M.K., Paragi, Z., Röttgering, H. et al., 2013, MNRAS, 431, L58

[11] Yuan, F., Yu, Z., Ho, L.C., 2009, ApJ, 703, 1034

[12] Yuan, F., Cui, W., 2005, ApJ, 629, 408 